\title{
Nuevos escenarios para la conversación política: el uso de WhatsApp por parte de los partidos durante la campaña electoral de abril de 2019 en España
}

\section{Rocío Zamora Medina ${ }^{1}$ José Carlos Losada Díaz²}

Recibido: 13/01/2020

Aprobado por pares: 06/02/2020
Enviado a pares: $13 / 01 / 2020$

Aceptado: 07/02/2020

DOI: 10.5294/pacla.2021.24.1.2

Para citar este artículo / to reference this article / para citar este artigo Zamora, R. y Losada, J. C. (2021). Nuevos escenarios para la conversación política: el uso de WhatsApp por parte de los partidos durante la campaña electoral de abril de 2019 en España. Palabra Clave, 24(1), e2412. https://doi.org/10.5294/pacla.2021.24.1.2

\section{Resumen}

La democratización de la tecnología ha permitido que los ciudadanos y los partidos políticos encuentren nuevos caminos para el diálogo y la conversación social. De hecho, los líderes políticos "comienzan a gobernar con el móvil”. Dentro de las diferentes opciones para este diálogo se encuentran las aplicaciones de mensajería instantánea, como WhatsApp, que se consolidan como una herramienta habitual en las estrategias de comunicación de partidos y candidatos. Sin embargo, no existe en el ámbito de la investigación académica un desarrollo teórico que ayude a entender este fenómeno. Para afrontar tal vacío, esta investigación incluye como principal objetivo tratar de analizar científicamente el uso que los partidos políticos otorgaron a estas herramientas durante la campaña para las elecciones generales en España en abril de 2019. Tomando como referencia los estudios previos relacionados con el uso de esta herramienta en contextos electorales en Bra- 
sil, la India o Nigeria, este trabajo se convierte en un estudio empírico pionero realizado en la primera "Elección de WhatsApp" en España, a partir de su protagonismo como red social con más de 25 millones de usuarios. La parte nuclear de esta investigación descansa en el análisis de contenido cualitativo de todos los contenidos creados en WhatsApp por los cinco principales partidos políticos (Partido Socialista Obrero Español, Partido Popular, Vox, Ciudadanos y Unidas Podemos) que concurrieron a estas elecciones. Esta investigación empírica y comparativa se centró en categorías específicas, como nivel de actividad, tipo de contenido, enlaces a otras redes sociales, propósito principal del contenido, presencia de contenido falso y negativo y nivel de interacción. Los resultados demostraron un diferente uso de esta herramienta por parte de los partidos políticos españoles, siendo los partidos tradicionales los que presentaron un mayor nivel de actividad. Además, si bien los contenidos audiovisuales fueron un recurso frecuente, la campaña española no estuvo marcada por una difusión agresiva de contenido falso.

\section{Palabras clave (Fuente: tesauro de la Unesco)}

WhatsApp; redes sociales; comunicación política; campañas digitales; elecciones. 


\section{New Settings for Political Discussion: Parties Using WhatsApp during the April 2019 Electoral Campaign in Spain}

\section{Abstract}

The democratization of technology has allowed citizens and political parties to find new avenues for dialogue and social debate. Actually, political leaders "have begun to govern with their mobiles." Among the multiple options for dialogue, instant messaging applications, such as WhatsApp, have grown firm as a standard tool for parties' and candidates' communication strategies. Nonetheless, there is no theoretical approach from academic research that helps to understand this phenomenon. Consequently, this research attempts to scientifically analyze how political parties in Spain employed these tools during the campaign for the general elections in April 2019. Building on previous studies related to the use of this microblogging in electoral contexts in Brazil, India, or Nigeria, this pioneering empirical study focuses on the first "WhatsApp election" in Spain due to its role as a leading social network with over 25 million users. This research's core lies in the qualitative analysis of all the content created on WhatsApp by the five major political parties (Partido Socialista Obrero Español, Partido Popular, Vox, Ciudadanos, and Unidas Podemos) that participated in these elections. This empirical and comparative research addresses specific categories, such as activity level, content type, links to other social media, the primary purpose of the content, fake and harmful content, and interaction level. Results showed a different use of this tool by Spanish political parties, with traditional parties showing the highest level of activity. Furthermore, although audiovisual content was a frequent resource, the Spanish campaign was not marked by an aggressive dissemination of false content.

\section{Keywords (Source: Unesco Thesaurus)}

WhatsApp; social networks; political communication; online campaigning; elections. 


\section{Novos cenários para o diálogo político: 0 uso do WhatsApp pelos partidos durante a campanha eleitoral de abril de 2019 na Espanha}

\section{Resumo}

A democratização da tecnologia permite que os cidadãos e os partidos políticos encontrem novos caminhos para o diálogo social. De fato, os líderes políticos "começam a governar com o celular". Dentro das diferentes opções para esse diálogo, encontram-se os aplicativos de mensagem instantânea, como WhatsApp, que são consolidados como ferramenta habitual nas estratégias de comunicação de partidos e candidatos. Contudo, não existe, no âmbito da pesquisa acadêmica, um desenvolvimento teórico que ajude a entender esse fenômeno. Para preencher essa lacuna, esta pesquisa inclui como principal objetivo analisar cientificamente o uso que os partidos políticos outorgaram a essas ferramentas durante a campanha para as eleições gerais na Espanha em abril de 2019. Tomando como referência os estudos prévios relacionados com o uso desse microblogging em contextos eleitorais no Brasil, na Índia e na Nigéria, este trabalho se converte no primeiro estudo empírico realizado na primeira "eleição do WhatsApp" na Espanha, a partir de seu protagonismo como primeira rede social com mais de 25 milhões de usuários. A parte nuclear desta pesquisa está na análise de conteúdo qualitativo de todos os conteúdos criados no WhatsApp pelos cinco principais partidos (Partido Socialista Obrero Español, Partido Popular, Vox, Ciudadanos e Unidas Podemos) que participaram dessas eleições. Nesta pesquisa empírica e comparativa, centrou-se em categorias específicas, como nível de atividade, tipo de conteúdo, links a outras redes sociais, objetivo principal do conteúdo, presença de conteúdo falso e negativo, e nível de interação). Os resultados mostraram um uso diferenciado desta ferramenta pelos partidos políticos espanhóis, com os partidos tradicionais apresentando o maior nível de atividade. Além disso, embora o conteúdo audiovisual fosse um recurso frequente, a campanha espanhola não foi marcada por uma difusão agressiva de conteúdos falsos.

\section{Plavras-chave (Fonte: tesauro da Unesco)}

WhatsApp; redes sociais; comunicação política; campanhas digitais; eleições. 


\section{Introducción}

La comunicación política ha estado marcada durante años por la unidireccionalidad propiciada por las características propias de los medios de comunicación masivos. Era un esquema sencillo y controlable por parte de los partidos. Los políticos lanzaban indiscriminadamente sus mensajes a través de los medios de comunicación con espacios pagados o contenidos informativos, y los medios, con sus correspondientes filtros ideológicos, cuando los había, se encargaban de traspasarlos a los ciudadanos, que se convertían en meros receptores pasivos de todos estos mensajes. No había opciones, o muy residuales, de que estos ciudadanos pudieran expresar su opinión o interactuar directamente con los políticos y sus partidos (Jenkins, 2006).

La llegada de internet, y en particular del universo 2.0, propició el nacimiento de espacios públicos de comunicación en que cualquier usuario puede no solo reaccionar, sino también interactuar, compartir, difundir e, incluso, crear contenido de carácter político (Gutiérrez-Rubí, 2019a). Como destaca Maarek (2014), todo esto puede hacerse sin ningún tipo de limitación para los usuarios, tanto en la diseminación de mensajes como en su viralidad. Por ello, el entorno digital promovido por la introducción de la web 2.0 ha acabado aportando una nueva forma de comprender la comunicación política que ha introducido en el debate público y político conceptos como participación, diálogo, apertura, transparencia y bidireccionalidad, entre otros (Giansante, 2015).

Esta nueva situación ha modificado claramente ciertos parámetros de la comunicación política que se pensaba serían permanentes. Por lo que respecta a los ciudadanos, estos han alcanzado un nivel mucho más elevado de empoderamiento respecto de los asuntos públicos; han condicionado en alguna medida la agenda pública, las opiniones de los candidatos y hasta las decisiones de los dirigentes, y han llegado a representar una suerte de vigilancia activa dentro del mundo virtual. En un contexto de crisis social y política, los ciudadanos, desencantados con las instituciones públicas, han tratado de ejercer un control sobre los asuntos públicos practicando una for- 
ma de tecnopolítica que nace para aunar las características del movimiento de regeneración política y las herramientas tecnológicas (Gutiérrez-Rubí, 2014). Por otro lado, los partidos políticos han visto cómo, gracias a estas herramientas sociales, pueden dejar de depender de modo tan evidente de los medios de comunicación de masas, de sus filtros o de sus peajes, para poder difundir sus mensajes (Castells, 2009; Jenkins, 2006), lo que han querido aprovechar para encontrar y explotar nuevas formas de comunicación con el electorado (Penteado, 2011). Esto explica que todos ellos utilicen en alguna medida herramientas 2.0 , principalmente redes sociales y mensajería electrónica y, con ello, modifican la estrategia de comunicación acostumbrada hasta ese momento (Giansante, 2015).

Una de las herramientas propias de este universo 2.0 que con más éxito han sido utilizadas en estos últimos años son las aplicaciones de mensajería electrónica, cuyas características hacen posible avanzar significativamente en el ámbito de la comunicación política online. Algunos autores llegan a asegurar que "tienen el potencial de contribuir a cambios fundamentales en la participación política” (Martín, 2014, p. 189). Y esto ocurre, en primer lugar, por la enorme cantidad de usuarios que se han descargado este tipo de aplicaciones y las usan para todo tipo de actividades profesionales y personales. De hecho, en este momento, estas herramientas de mensajería electrónica se han convertido en "el principal medio de acceso a internet en España” (Varona-Aramburu et al., 2017, p. 642).

\section{El potencial de WhatsApp como herramienta de conversación social}

WhatsApp cuenta, en la actualidad, con más de 1500 millones de usuarios activos en el todo el mundo (Sydow, 2019). A pesar de haber tenido fuertes competidores en el campo de la mensajería, como QQ Telegram o Wechat, que ofrecían más y mejores servicios, la app ha sabido adaptarse y ha ido incluyendo paulatinamente entre sus servicios todo aquello que sus competidores ofrecían por separado. De esta forma, ha logrado consolidarse como la red social y de mensajería instantánea más utilizada del mundo (20minutos, 2019). 
En España, según el Estudio Anual de Redes Sociales 2019, existían 25 millones de usuarios de WhatsApp. Esto quiere decir que más de la mitad de la población tiene descargada esta aplicación en su dispositivo móvil (Serrano, 2019). Además, el nivel de conocimiento sobre esta aplicación entre la población es del 93 \% y la valoración de uso es de un 8,5 (la app más valorada). "No utilizarla es quedarse fuera y renunciar a estar allá donde está el electorado" (Gutiérrez-Rubí, 2015), y esto es algo que los partidos políticos han sabido detectar, tratando de impulsar sus canales de WhatsApp como novedad ante las elecciones generales de abril de 2019 (Serrano, 2019), objeto principal de este artículo.

Gutiérrez-Rubí (2019b) enumera muchas de las ventajas que esta herramienta posee para su uso político. Entre ellas, el autor destaca que "WhatsApp permite una relación de persona a persona, entre iguales. Eso valida el mensaje, le da interés y credibilidad”. El canal también concede cierto tono "clandestino", que "motiva un sentido de pertenencia". No son solo estas las ventajas. Para Gutiérrez-Rubí (2019b), la aplicación cuenta con otras ventajas como "la conexión permanente". De este modo, "los mensajes te llegan automáticamente y eso es más cómodo”. Además, "no hay una exposición pública para quien lanza el mensaje, como sí ocurre en Facebook, y no te obliga a mostrar ese contenido a gente que no comparte tus ideas. Se alcanza un nivel casi de intimidad y no deja rastro". Por último, los mensajes de WhatsApp permiten "una gran plasticidad, una personalización de emojis, memes, fotos y vídeos", lo que hace el mensaje "mucho más atractivo”.

Por todo ello, la utilización de esta herramienta con fines políticos resulta más que evidente. Por una parte, WhatsApp facilita la difusión de mensajes y permite de forma sencilla que los usuarios amplíen el alcance reenviando la información recibida en cualquier formato, lo que explica su enorme capacidad de viralización gracias a su facilidad para segmentar y reenviar mensajes a grupos concretos (Jaume, 2015). WhatsApp se convierte así en un canal interactivo y cercano al usuario, cuyas respuestas a tiempo real van a conseguir establecer un vínculo comunicativo sólido que permitirá "maximizar la capacidad de movilización". Todo ello, unido a su elevada 
capacidad para ampliar las bases de datos de los emisores, dinamizar a los prescriptores y distribuir contenidos mediante enlaces para compartir publicaciones desde redes sociales (Barredo, 2019).

El principal inconveniente de WhatsApp, sin embargo, es su enorme capacidad para fomentar la difusión de información falsa, bulos, rumores y fake news. Según las investigaciones del movimiento ciudadano Avaaz difundidas por la Agencia EFE (20minutos, 2019), el $36 \%$ de los usuarios de WhatsApp ha recibido en el último mes mensajes de contenido político o ideológico. El estudio aseguraba, además, que hasta 10 millones de ciudadanos perciben bulos o textos de odio por esta red social. Con estos datos, WhatsApp se convierte en la segunda plataforma de "desinformación y contenido de odio”, por detrás de Facebook.

Estos datos son la manifestación de una evidencia muy clara: el uso de WhatsApp como una herramienta perfecta para la difusión de todo tipo de información falsa, rumores o bulos, es decir, un instrumento que por sus características es ideal para provocar y gestionar campañas de desinformación altamente viralizables a través de las redes de confianza de nuestros teléfonos móviles. Esta capacidad, junto con el uso masivo por parte de la población, ha sido un mecanismo muy útil para los partidos políticos y candidatos en todo el mundo, en especial, en citas electorales.

\section{Precedentes internacionales del uso de WhatsApp en contextos políticos electorales}

WhatsApp ya ha sido utilizado como herramienta político-electoral en diferentes países, a pesar de su carácter innovador. En este sentido, consideramos pertinente considerar estos ejemplos previos como antecedentes del trabajo que aquí se presenta.

\section{Nigeria}

WhatsApp es la aplicación de mensajería más popular en 40 países africanos (Cheeseman, 2019), entre los que se incluye Nigeria. Allí los dos candidatos principales en las elecciones de 2019, el presidente Muhammadu 
Buhari y el líder de la Oposición Atiku Abubakar, contrataron equipos completos para generar y enviar mensajes a través de medios sociales. La estrategia de Buhari tenía un mayor presupuesto y fue particularmente exitosa. Estableció una red de representantes locales y regionales conectados a un "comando central" en Abuya. Esto permitió que la campaña enviara rápidamente mensajes del nivel nacional al local, al tiempo que respondía a mensajes hostiles y rumores compartidos por sus rivales. Por lo que respecta a la oposición, los líderes contaron con una forma barata de responder a los mensajes gubernamentales y para coordinar campañas anticorrupción y, de ese modo, fortalecer la democracia interna en el país.

\section{Colombia}

En las elecciones presidenciales en Colombia de 2018 el partido de Iván Duque, candidato que finalmente se hizo con la Presidencia, había articulado una red de grupos de WhatsApp a través de los cuales realizaba encuestas segmentadas geográficamente para recapitular información acerca de las demandas de la población y, a partir de ellas, el candidato elaboraba un discurso con propuestas hechas a la medida de sus problemas. Gutiérrez-Rubí (2015) advertía cómo este caso mostraba la facilidad con las que las informaciones falsas podían ser distribuidas por WhatsApp, pasando, en ocasiones, desapercibidas por el usuario que actuaba, además de como receptor, como difusor de esta.

\section{La India}

WhatsApp es también la app más descargada en la India, con más de 200 millones de usuarios activos, que abarca el $20 \%$ del total de usuarios de la aplicación en el mundo (Singh, 2017). Este dato cuantitativo la convertía en una herramienta jugosa para la clase política. A principios de 2017, antes de las elecciones para Uttar Pradesh, Bhartiya Janta Party (BJP) empleó WhatsApp de forma masiva para la movilización, la coordinación y el acercamiento a los votantes. Para ello, formó 10344 grupos de WhatsApp, solo para coordinar y hacer circular los medios entre sus trabajadores del partido (Verma, 2017). No se pudo detener la difusión de desinformación y noticias falsas, que se convirtió en el principal desencadenante de disturbios de Muzaffarnagar en Uttar Pradesh, solo ocho meses antes de las eleccio- 
nes federales. Este uso desinformativo de la app llegó también a influir en las tensiones entre la India y su vecino Pakistán, que propició en 2019 una verdadera guerra de información multiplataforma y que alcanzó también a los medios de comunicación.

Antes de las elecciones de 2019, la desinformación viral y la propaganda alcanzaron una nueva dimensión. El Computational Propaganda Research Project (ComProp) (Oxford Internet Institute, 2019) descubrió que, entre mediados de febrero y la víspera de la primera ronda de votación, la proporción de noticias políticas polarizadas e información en circulación en las redes sociales en la India era peor que todos los otros estudios de casos de países analizados. Los investigadores descubrieron que más de una cuarta parte del contenido compartido por BJP de Modi era basura, al igual que una quinta parte de la información compartida por el opositor Congreso Nacional Indio. Ante estos hechos, WhatsApp decidió cerrar 400000 cuentas en el país cada mes. La compañía también se gastó US $\$ 10$ millones en una campaña de educación pública sobre los peligros de la desinformación (Financial Times, 2019). Este mismo periódico calificó el voto de este año de "Elección de WhatsApp" de la India, de forma similar a Brasil. Además, WhatsApp limitó el tamaño de los grupos y su función de reenvío. En preparación para las elecciones, agregó una "línea de aviso" que permitía a los usuarios informar y verificar posibles noticias basura.

\section{Brasil}

Una de las referencias internacionales más importantes sobre el uso de WhatsApp en política tuvo lugar en Brasil en las elecciones presidenciales celebradas en octubre de 2018. En estos comicios, el candidato del Partido Social Liberal, Jair Bolsonaro, venció con el 55,21 \% de los votos a su adversario, el candidato del Partido de los Trabajadores, Fernando Haddad, que obtuvo el 44,79\% de los votos (Galarraga, 2018). Muchos consideran que estos comicios representan la consolidación de WhatsApp como la herramienta de propaganda política por excelencia (Borella, 2018). Tanto es así que los seguidores de Bolsonaro habían asumido que WhatsApp había sido clave para el triunfo de su candidato y en su investidura, meses después, co- 
menzaron a corear el nombre de la app delante de los medios de comunicación que cubrían el acto (Nogueira, 2019).

En Brasil, 6 de cada 10 habitantes utilizan WhatsApp para comunicarse (Digital News Repport, 2018). Los partidos políticos supieron aprovechar la oportunidad que les ofrecía la red de mensajería instantánea para poder llevar a cabo una campaña de comunicación sin precedentes (Hazard, 2018). Aunque ambos partidos utilizaron este canal, lo cierto es que los seguidores de Bolsonaro eran más y estaban mejor organizados, algo que explica por qué WhatsApp resultó ser determinante para lograr su victoria en las urnas (Oliveira, 2018). Según Estudio Datafolha (2018), el $40 \%$ de los votantes de Bolsonaro difundieron información política en WhatsApp durante la campaña electoral, frente al $22 \%$ de los votantes de Haddad. Aunque ambos candidatos habían sido víctimas de memes y bulos, la campaña contra Fernando Haddad y el Partido de los Trabajadores fue más agresiva (Oliviera, 2018). Existen datos alarmantes sobre el volumen de información falsa al que estuvieron sometidos los usuarios de WhatsApp brasileños antes de la celebración de las elecciones. De hecho, tan solo 4 de las 50 imágenes analizadas (el $8 \%$ ) que fueron distribuidas en 347 grupos de WhatsApp eran verdaderas (Tardáguila et al., 2018).

Estas evidencias sobre la difusión indiscriminada de informaciones falsas acabó provocando una oleada de denuncias hacia la compañía (Vosoyghi et al., 2018), que se vio obligada a intervenir en la campaña limitando el número de integrantes de los grupos, el número de veces que se pueden reenviar los mensajes e, incluso, llegó a cerrar las cuentas de miles de personas, entre ellas, el senador Flavio Bolsonaro, hijo del candidato a la presidencia Jair Bolsonaro. WhatsApp alegó que el motivo que les movió a eliminar de forma tajante numerosas cuentas de usuarios fue el comportamiento sospechoso de estos, en relación con la emisión de mensajes que incitan al odio, si son ofensivos o el número de veces que se repiten, además de considerar el número de bloqueos que ha recibido un usuario por parte de otros (Senra, 2018). No obstante, según algunos autores advierten, es muy difícil que se puedan investigar ciertos comportamientos políticos propiciados por el mal uso de WhatsApp (Gragnani, 2018). 


\section{Metodología}

El objetivo principal de esta investigación ha sido analizar el uso de WhatsApp realizado por los partidos políticos españoles como herramienta innovadora de comunicación electoral. Para ello, se ha llevado a cabo un estudio descriptivo que, por un lado, incluye un análisis cuantitativo de la actividad llevada a cabo por los cinco principales partidos políticos que concurrían a las elecciones generales de 2019: Partido Popular (PP), Ciudadanos (C's), Vox, Partido Socialista Obrero Español (PSOE) y Unidas Podemos (UP), en sus canales oficiales de WhatsApp, además de un análisis cualitativo pormenorizado de la información publicada en dichos canales.

Para la obtención de los datos, se optó por la técnica de observación sistemática participante en la que el investigador establece previamente una serie de categorías de observación a partir de las cuales realiza la investigación empírica. La principal ventaja de esta técnica consiste en que permite la observación directa al permanecer integrado en el medio ambiente del grupo estudiado y tener la posibilidad de intervenir en sus prácticas e interpretar activamente sus conductas más significativas. Esta decisión nos obligó a darnos de alta en los canales de estos partidos.

Concretamente, la captación de usuarios que llevaron a cabo los partidos políticos para ampliar sus bases de datos desde sus perfiles en WhatsApp propició la creación de listas de difusión, un procedimiento bastante similar en todos los partidos estudiados, aunque con algunas diferencias. Así, mientras que en el canal oficial de WhatsApp de PSOE y Vox bastaba con enviar la palabra "alta" para realizar el registro, tanto en el PP como UP se exigía el lugar de procedencia del usuario para el registro. En el caso de C's, los usuarios interesados en recibir información tenían que darse de alta en el canal a través de unos pasos establecidos por el partido.

El acceso a los canales oficiales de WhatsApp de los partidos políticos no estuvo exento de una serie de problemas e incongruencias. Fue necesario seguir un proceso estándar consistente en asociar el nombre de cada partido político con WhatsApp en Google. Mientras que para el caso de C's y UP la búsqueda nos dirigía directamente al enlace con la infor- 
mación correcta, cuando realizamos la búsqueda en Google de los términos "PP + WhatsApp", el buscador nos dirigió al espacio de la web oficial del partido, pero el número que facilitaban no era el que habían difundido a través de redes sociales con el hashtag \#VenteAlPP, el cual se encontraba en el dominio vente.pp.es. Finalmente, este error fue subsanado, por lo que los usuarios dejaron de inscribirse en un canal que durante la campaña electoral estaba en desuso (Gutiérrez-Rubí, 2019). Por otra parte, el acceso al número de WhatsApp del PSOE fue también complicado desde su propia web y solo pudo obtenerse a través del grupo de WhatsApp del partido a nivel local.

Las fechas establecidas para la recogida de datos abarcaban desde el 12 de abril (día en el que comenzó la campaña electoral) hasta el 29 de abril (el día después de la celebración de las elecciones generales). Esta limitación temporal permitió conocer, por un lado, el nivel de uso de esta nueva herramienta de comunicación política durante las fechas de la campaña electoral oficial y la actividad realizada durante la jornada de reflexión, con sus condicionantes legales, para comprobar si era o no respetada por los partidos. Finalmente, también se consideró oportuno analizar la actividad desarrollada el día de las elecciones y el día después de conocer los resultados electorales. Una vez realizada la identificación de las unidades de observación, se llevó a cabo un registro detallado y diario de la totalidad de los mensajes que enviaron cada uno de los partidos a través de su canal oficial de WhatsApp a sus suscriptores durante las fechas del estudio, con el fin de obtener los datos necesarios para nuestra investigación. El registro de estos se efectuó a través de la cumplimentación de una serie de tablas específicas que incluían las categorías previstas para obtener la información pertinente. Concretamente, el análisis se centró en la identificación de la fecha y hora de publicación de estos post, así como el contenido textual y visual propio de cada mensaje, además de comprobar si estos enlazaban o no con otras redes sociales.

\section{Resultados}

Como primer resultado de la investigación, el análisis cuantitativo que resume la actividad global desarrollada por parte de los partidos políticos en 
sus canales oficiales de WhatsApp durante la campaña electoral de 2019 en España demostró notables diferencias, tal y como se muestra en la tabla 1.

\section{Tabla 1. Actividad de los partidos políticos en sus canales oficiales de WhatsApp}

\begin{tabular}{|c|c|c|c|c|c|c|}
\hline (6) & (PP) & Ciudadanos & 留 & PSOE & $\begin{array}{l}\text { UNIDOS } \\
\text { PODEMOS. }\end{array}$ & \\
\hline Número de mensajes diarios & $1-8$ & $1-2$ & & $1-4$ & 1 & 1 \\
\hline Número de enlaces a otra red social & 39 & 1 & & 21 & 4 & 1 \\
\hline $\begin{array}{l}\text { Número de enlaces hacia un medio } \\
\text { de comunicación }\end{array}$ & 6 & 0 & & 5 & 0 & 0 \\
\hline $\begin{array}{l}\text { Número total de mensajes durante } \\
\text { la campaña }\end{array}$ & 38 & 5 & & 19 & 4 & 1 \\
\hline
\end{tabular}

Fuente: elaboración propia.

Así, los denominados partidos tradicionales, como el PP, que llegó a emitir hasta 38 mensajes durante el tiempo analizado, o el PSOE, que alcanzaba los 19 mensajes, fueron considerablemente más activos en su canal de WhatsApp en comparación con los partidos nuevos, tales como C's, con cinco mensajes; UP, con cuatro, y en menor medida, Vox, con tan solo un mensaje. En lo que se refiere a número de mensajes diarios emitidos, también fueron los dos partidos tradicionales, PP y PSOE, los que más hicieron uso de la red de mensajería instantánea por excelencia, que alcanzaron cifras de hasta ocho mensajes diarios en el primer caso y cinco en el segundo. El resto de los partidos se conformaron con un solo mensaje emitido al día.

Un aspecto interesante en relación con el uso de WhatsApp en contextos electorales es hasta qué punto se trata de una herramienta que está siendo utilizada para que los partidos integren su estrategia de campaña online a través del enlace con otras redes sociales. En este sentido, la investigación realizada demostró que esta práctica fue utilizada por todos los partidos, pero de un modo destacado una vez más por el PP y el PSOE. Estos dos partidos fueron también los únicos que incluyeron en sus mensajes enlaces a publicaciones de medios de comunicación tradicionales. 
Una vez ofrecidas las cifras generales de actividad por parte de los partidos políticos en WhatsApp, se ofrecen los resultados más significativos del análisis cualitativo del contenido de dichos mensajes y algunas curiosidades particulares del lanzamiento de la herramienta por parte de cada partido.

\section{Partido Popular}

El uso de WhatsApp como herramienta de comunicación política por parte del PP ha estado determinado por una serie de características comunes. Por un lado, el contenido de los mensajes (38) obedecía a una estrategia que centraba la atención en los actos de campaña de su candidato, Pablo Casado, rodeado de multitudes y, en algunas ocasiones, también acompañado de su esposa. No obstante, también se utilizó esta herramienta para difundir actos de agenda y algunas medidas incluidas en su programa electoral, incluso, para lanzar mensajes de ataque contra el presidente del Gobierno, Pedro Sánchez. Sin embargo, el contenido textual no fue lo más destacable, ya que en la campaña del partido ha predominado el material audiovisual. A través de WhatsApp, el PP remitió, en numerosas ocasiones, a enlaces a vídeos de su canal en YouTube, que, en su mayoría, no sobrepasaban los dos minutos de duración, y que podían reproducirse directamente dentro de la misma app. Tan solo hubo dos ocasiones en las que el vídeo no procedía de YouTube. Por un lado, el vídeo de apenas un minuto del expresidente del Gobierno, José María Aznar, ofrece un discurso en un ambiente distendido durante la celebración de una reunión de amigos. Por otro, el vídeo que difundió el partido tras conocer las encuestas del Centro de Investigaciones Sociológicas (CIS) que vaticinaban la victoria del PSOE.

Uno de los vídeos de YouTube que tuvo mayor repercusión, y que de hecho se convirtió en trending topic en Twitter, fue con el que iniciaron la campaña electoral titulado Falcón Viajes y creado por las Nuevas Generaciones del Partido Popular. En él se valían del humor y de la ironía para criticar el uso que hacía el presidente del Gobierno de los medios de transporte oficiales. Con 35516 reproducciones, el Falcon Viajes fue el vídeo con más visualizaciones en YouTube de todos los que ha compartido el PP 
a través de WhatsApp, seguido del vídeo explicativo en el que Narciso Michavila se vio obligado a desmentir el bulo que había circulado en redes sociales acerca del método de votación $1+1+1$ para el Senado. En este vídeo, que alcanzó las 23256 visualizaciones, se pedía repartir el voto entre los tres partidos de la derecha, para "evitar regalarle el Senado a Pedro Sánchez”, algo que, paradójicamente, afectaría de manera negativa a la derecha. Además, muchos de estos vídeos de YouTube lanzados a través del canal de WhatsApp del PP remitían indirectamente a Twitter, al incluir los lemas de la campaña y los creados en especial para representar el contenido del mensaje o del vídeo de YouTube mediante hashtags.

En lo referente al uso de la segmentación territorial para difundir mensajes específicos, pudimos comprobar cómo, tras darnos de alta en el canal de WhatsApp para una comunidad autónoma, el PP envió dos mensajes específicos para los ciudadanos de la región de Murcia. El primero fue el vídeo con el que presentaban al candidato que lideraba la lista del PP para el Congreso de los Diputados por Murcia y número dos de la formación política a nivel nacional, Teodoro García, y el segundo, la imagen con la información de agenda para comunicar el acto de campaña del PP en Murcia, en el cual intervendrían Pablo Casado, Teodoro García, Fernando López Miras (presidente de la Comunidad Autónoma de Murcia) y José Ballesta (alcalde de la ciudad de Murcia).

Por otra parte, los mensajes enviados coincidentes con la celebración del debate de A3Media, introdujeron dos estrategias nuevas: la inclusión de enlaces a medios de comunicación, para que el usuario cumplimentara la encuesta acerca del ganador del debate y el enlace a contenidos de la web http://biblioteca.pp.es a través de montajes con los que pretendía "desmentir las mentiras de Pedro Sánchez durante el debate” para los que se pedía su difusión.

Por su parte, el lenguaje utilizado en los mensajes textuales se basó en mensajes breves en los que, en ocasiones, se incluían apelaciones directas al receptor como el “Y tú ite unes?”. Además, dentro de la estética del mensaje, también han tenido cabida los característicos emoticonos de WhatsApp 
que aportaban la nota informal a los textos, como los utilizados en el mensaje en el que una serpiente representaba a Torra, Puigdemont y Otegui (peligro); el corazón roto que acompañaba a la frase "liquidando nuestro país" (dolor), o el uso del avión que simbolizaba "la utilización de las instituciones”, argumento repetido durante la campaña para denunciar el uso que Sánchez hace de los medios de transporte oficiales.

En definitiva, el PP aprovechó al máximo las características que WhatsApp ofrece como nueva herramienta de comunicación política, integrándose tanto dentro de la estrategia online como de la offline. La actividad del canal fue constante hasta el 25 de abril, día en el que WhatsApp cerró la cuenta oficial del partido por incumplir las normas de la compañía. Sorprendentemente, el partido utilizó las listas de difusión para enviar dos mensajes de texto (sms) a todos los números de los usuarios registrados en ellas el día previo a la jornada de reflexión. Ambos mensajes incluían enlaces a vídeos de YouTube: en el primero, invitaban a la reflexión “El \#28A piensa bien tu voto” y, en el segundo, apelaban a la unidad "El voto nos une, únete”.

\section{Partido Socialista Obrero Español}

En actividad del canal oficial del PSOE durante la campaña electoral en WhatsApp, fue también destacable (19 mensajes), a pesar de que hubo días en los que el partido no emitió ningún mensaje. El principal uso que el PSOE dio a la herramienta fue utilizarla como plataforma para redirigir a los usuarios hacia sus cuentas oficiales de Twitter (incluyendo en algún mensaje la oficial de Pedro Sánchez), sin duda, la red social más utilizada, y Facebook, así como a otras redes sociales como Spotify. A través de esta última red social compartieron la playlist oficial de la campaña del partido, en la cual había canciones como Cayetano, de Carolina Durante, o Señoras bien, de Las Bistecs, vinculadas con estereotipos negativos de la derecha. De esta forma, el PSOE conseguía que los usuarios accedieran desde su dispositivo móvil a las cuentas oficiales del partido en redes. Solo en una ocasión el WhatsApp del partido remitía a la web del partido y a varios medios de comunicación, para que el usuario realizara la encuesta sobre quién había ganado el debate de A3Media. 
La campaña del PSOE en WhatsApp también ha sido una campaña marcada por el contenido audiovisual. La mayoría de los vídeos publicados en diferentes redes sociales y difundidos a través de la red de mensajería instantánea tenían una duración que oscilaba entre 30 segundos y 1 minuto. Cabe destacar que, independiente de que el contenido del vídeo fuese un spot en el que participaban actores que recreaban las situaciones que marcaba el discurso o la escenificación del mensaje narrado a través de ilustraciones animadas, el cierre de estos vídeos tenía elementos en común: el primer plano de Pedro Sánchez en blanco y negro repitiendo los lemas del partido durante la campaña o los mismos plasmados sobre fondo rojo con letras blancas.

En este caso, el candidato, Pedro Sánchez, fue el protagonista de uno de los vídeos difundidos a través de WhatsApp en el que se publicaba un extracto del mitin del partido celebrado en Palma de Mallorca. El vídeo con mayor número de reproducciones alcanzó las 29000 reproducciones en Twitter, pero no consiguió estar dentro de los primeros puestos del trending topic nacional.

Por lo que se refiere a los contenidos específicos que se emitieron, el partido difundió mensajes positivos y movilizadores, que apelaban a los sentimientos de la sociedad, a sus preocupaciones y al fomento del voto, para evitar que la extrema derecha llegase al poder de la mano del centro-derecha que sustentaba su lema de campaña "La España que quieres". El PSOE aprovechó también la herramienta para difundir algunas de sus propuestas electorales, incluso el propio programa electoral en PDF, así como informar sobre actos programados durante la campaña o relativos a los debates electorales. Sin embargo, a diferencia otros partidos, el PSOE no incluyó entre sus mensajes de WhatsApp ningún ataque directo a otras formaciones.

En cuanto a la redacción de los mensajes, en su mayoría incluían un lenguaje cercano, con expresiones como "Buen día!", repleto de hashtags con los lemas de la campaña, y cargado de mensajes que transmitían ilusión y esperanza. También se incluyeron emoticonos como la rosa (sím- 
bolo representativo del partido), el puño cerrado, la urna con el sobre del voto, el corazón rojo e, incluso, la bandera de España. Este último emoticono fue utilizado en numerosas ocasiones para ilustrar el sentimiento patriótico predominante.

La petición de reenvío y el proceso que el usuario debía seguir para darse de baja del canal también fueron incluidos dentro del cuerpo textual de algunos mensajes. Incluso enviaron un mensaje con un enlace que remitía a la política de privacidad del canal de WhatsApp del partido localizada dentro de su página web. De esta forma, el usuario estaba al tanto de cómo dejar de recibir mensajes de la formación política en cualquier momento.

Finalmente, la campaña en WhatsApp acabó el 25 de abril, cuando la empresa cerró la cuenta del PSOE alegando los mismos motivos que con el resto de formaciones que conforman nuestra muestra: incumplimiento de las normas de uso de la compañía.

\section{Unidas Podemos}

Resulta llamativo el uso casi imperceptible que hizo UP de WhatsApp, a pesar de ser el partido más activo en redes sociales. En sus únicos cuatro mensajes difundidos, el uso principal que hizo este partido de esta herramienta de comunicación política se sustentó en compartir enlaces que remiten a las cuentas oficiales de la formación política en otras redes sociales, tales como Facebook o YouTube, con la peculiaridad de que la previsualización permitía al usuario poder reproducir los vídeos dentro de la misma $a p p$, sin necesidad de dirigirse a la red social de la que provenían.

El contenido de estos vídeos, algo más extensos que los de otros partidos, y la forma de representar el mensaje han sido variados. La situación de los autónomos narrada y representada a través de ilustraciones, el spot de UP para la campaña electoral, la intervención de Irene Montero en el programa de La Sexta Noche (vídeo que llegó a alcanzar las 745000 visualizaciones) y la explicación que daba el diputado de Podemos, Alberto Rodríguez, sobre el coste de la luz conforman el contenido de los mensajes difundidos 
por UP durante su campaña en WhatsApp. El corte del programa de La Sexta donde intervino Irene Montero, compartido en Facebook y difundido a través de WhatsApp, fue el vídeo con más visualizaciones de todos los que han circulado por su canal oficial, con 745000 visualizaciones.

Paradójicamente, aunque UP también pedía información acerca del lugar de procedencia durante los pasos a seguir para darnos de alta en su canal oficial, nunca llegó a enviar un contenido informativo específico a nivel territorial, algo que sí hizo el PP.

Tal y como sucedió con el resto de formaciones, WhatsApp cerró el canal oficial del partido el 21 de abril por incumplir la normativa de la compañía, dos días antes que en el resto de los otros partidos políticos, algo que no estuvo exento de polémica. Fue la propia formación la que denunció el "injusto" cierre de su cuenta alegando que realizaban el mismo uso de esta herramienta que el resto de partidos políticos, cumpliendo con todos los requisitos de la empresa y que contaban con la suscripción voluntaria y el consentimiento expreso de 60000 personas (Barredo, 2019). Por su parte, la compañía alegó que se limitaba a aplicar la misma normativa que regulaba el uso de la aplicación en el mundo; el envío masivo de mensajes y los programas de terceros para automatizar los mensajes fueron los motivos del cierre. Tras el cierre de la cuenta de UP, WhatsApp volvió a reabrirla "por error" el 25, pero la compañía no tardó en recapitular y cerrarla de forma permanente el 26 de abril (20minutos, 2019).

\section{Ciudadanos}

Aunque el canal oficial de C's tampoco ha sido utilizado por la formación naranja con asiduidad, este partido destaca por ser el único que ha utilizado WhatsApp para llevar a cabo una comunicación interactiva, directa y personalizada con el usuario, quien determinaba el nivel de conversación con el canal. En cualquier caso, la respuesta por parte del partido era escueta y no instantánea.

En lo referente a la difusión de los contenidos, C’s solo envío un único mensaje durante toda la campaña que incluía un enlace de YouTube en el que se publicaba el vídeo oficial de la campaña. El vídeo, que 
trataba sobre su programa económico y tenía una duración de cincuenta segundos, estaba dividido en dos partes. En la primera, los protagonistas eran una serie de niños que aparecen animando a sus progenitores a ir a votar, alegando que estaban cansados de escuchar una serie de quejas relacionadas con la economía, el trabajo, la clase política, etc. La segunda parte del vídeo se centra en la figura del candidato, Albert Rivera, durante un acto de campaña, en el que no faltan las banderas de C's, España y la Unión Europea (UE). En él, Rivera menciona los lemas de la campaña: "Vamos a votar, vamos a ganar. ¡Vamos España! ¡Vamos Ciudadanos!”. Con 2864445 visualizaciones, este vídeo se convirtió en el más reproducido de todos los que han compartido en WhatsApp las formaciones políticas analizadas.

En este caso, el lenguaje utilizado por esta formación política en su canal de WhatsApp fue cercano y correcto. En cada interacción, se interesaban por el usuario al que se dirigían: querían saber su nombre, su lugar de procedencia, su opinión acerca del programa electoral, etc. Esto animaba a continuar con la conversación, aunque esta se prolongase durante varios días. Además, el lenguaje informal se concretaba en el uso de diferentes emoticonos a lo largo de los contenidos textuales.

Como en el resto de los partidos, C's también utilizó su canal de WhatsApp para remitir al usuario a la página web de su partido, con el fin de hacerle llegar mediante enlace su programa electoral completo. Más allá de estos casos puntuales, no se registró ninguna otra actividad de esta formación política, que se vio obligada a cerrar su canal oficial también el 25 de abril, por los mismos motivos por los que cerraron las cuentas de las demás formaciones.

\section{Vox}

En el caso de Vox, el uso de WhatsApp como herramienta de comunicación durante la campaña electoral pasó prácticamente desapercibido, a pesar de que sí fueron más activos durante la precampaña, tal y como se aprecia en la tabla 4. En el único mensaje que Vox envió durante la campaña, se pedía la difusión entre todos los contactos de WhatsApp del usuario al que va destinado. Además, se hacía alusión a los medios de comunicación y, 
más concretamente, a la televisión, asegurando que estos no se harán eco de las palabras de Santiago Abascal durante el acto del partido en Granada.

El mensaje de texto incluía un enlace con previsualización a un vídeo de YouTube, la red social a la que más remisiones han hecho desde su canal de WhatsApp tanto en precampaña como durante la campaña. El contenido audiovisual del vídeo mostraba imágenes de un acto de campaña celebrado en el Palacio de Congresos de Granada. Durante el transcurso del vídeo destacaba la figura de su candidato, Santiago Abascal, quien hacía referencia al malestar del partido por no haber podido participar en el debate de A3Media. El mensaje fue saboteado por un error que cometió un responsable del partido, quien envió por equivocación la estrategia que la formación política había marcado respecto del veto en ese debate para manifestar su malestar por la cancelación de su participación, información que fue posteriormente publicada en los principales medios.

El vídeo de YouTube que difundieron a través de WhatsApp tenía una duración de 1 minuto y 40 segundos, y tuvo 208482 visualizaciones. En lo referente al formato textual, el lenguaje utilizado en ese mensaje resultaba cercano, dentro de la corrección, y alberga un sentimiento patriótico y movilizador. Además, al igual que en otras formaciones políticas, se hacía uso de emoticonos, entre los que figuraba la bandera de España, un elemento que no ha faltado en ninguno de sus mensajes, así como el signo de la victoria con los dedos de la mano, el brazo sacando músculo o un megáfono para simbolizar difusión.

La cuenta oficial de Vox en WhatsApp también fue cerrada por la compañía el 25 de abril, por los mismos motivos por los que cerraron las cuentas de las demás formaciones.

\section{Conclusiones}

WhatsApp, junto con otras aplicaciones de mensajería electrónica, se ha consolidado como una herramienta de comunicación política que partidos y candidatos utilizan de forma ordinaria, especialmente en contextos electorales. Las experiencias de los últimos años en países como Nigeria, 
la India, Colombia o Brasil han permitido observar cómo la gestión estratégica de esta plataforma ha logrado que los mensajes de los partidos alcanzaran a una importante proporción de ciudadanos, gracias a las características propias de esta herramienta, como su elevado poder de alcance, de segmentación y de viralización, mucho mayor que los canales tradicionales y hasta de las redes sociales mayoritarias. Pero, al mismo tiempo, el uso de WhatsApp han traído una difusión incontrolada de información falsa, de rumores y de bulos, que se han multiplicado en los teléfonos móviles de los usuarios, incapaces de confirmar o comprobar la veracidad de la información que les iba llegando a través de sus contactos. Esta posibilidad ha sido utilizada como un arma valiosa por las diferentes opciones políticas para desacreditar a sus adversarios.

El potencial de WhatsApp como herramienta electoral quedó demostrado en la campaña de las elecciones generales de abril en 2019 en España. En estos comicios, todos los partidos mayoritarios utilizaron este instrumento de mensajería electrónica con fines electorales, aunque lo hicieron de forma desigual. Por una parte, los partidos mayoritarios históricos, PP y PSOE, mantuvieron una actividad permanente durante toda la campaña, con varios mensajes diarios y aprovechando la herramienta para enlazar con otras redes sociales del partido y también con medios de comunicación tradicionales. Por otro lado, los partidos de reciente creación, C's, UP y Vox, tuvieron una actividad mucho más reducida, apenas enlazaron sus contenidos con otras redes sociales y en ningún caso lo hicieron hacia otros medios de comunicación. Todos los partidos emplearon mensajes de elevado contenido audiovisual y ajustados a los estándares coloquiales y de cercanía habituales en este tipo de herramientas.

Sorprendentemente, en comparación con el uso de WhatsApp en otros países, la campaña española no estuvo marcada por una difusión agresiva de contenido falso o fake news. Pero, a pesar de ello, la actividad de los canales de WhatsApp de todos estos partidos políticos fue detenida por la compañía a partir del 21 de abril, apenas unos días antes de que finalizara la campaña. La razón esgrimida fue el incumplimiento de las normas de uso de esta que no permiten la mensajería masiva o la utilización de programas de terceros para automatizar los mensajes. 
En definitiva, el trabajo contribuye a la creciente comprensión de WhatsApp como una herramienta para practicar la relación de proximidad en una campaña online (Palomo y Sedano, 2018) y ofrece aportaciones a considerar para apreciar el potencial de esta herramienta para la conversación política en contextos electorales.

\section{Referencias}

Barredo, A. (2019, 18 de enero). WhatsApp supera a Facebook como plataforma social más popular en el mundo. La Vanguardia. https:// www.lavanguardia.com/tecnologia/20190118/454183092892/ WhatsApp-supera-facebook.html

Benidelli, T. (2017, 15 de marzo). La fiscalía brasileña pide investigar a toda la clase dirigente por corrupción. El País. https://elpais.com/internacional/2017/03/14/actualidad/1489524802_196077.html

Borella, G. (2018, 18 de noviembre). WhatsApp, nueva arma de manipulación electoral. La Nación. https://www.lanacion.com.ar/opinion/ WhatsApp-nueva-arma-de-manipulacion-electoralpolitica-y-medios-nid2192142

Castells, M. (2009). Comunicación y poder. Alianza.

Cheeseman, N. (2019, 30 de julio). The sophisticated ways WhatsApp was used by political operatives in Nigeria's 2019 elections. Quartz Africa.https://qz.com/africa/1678202/how-whatsapp-impacted-nigerias-2019-election/

Digital News Repport. (2018). Overview. http://www.digitalnewsreport.org/

El Confidencial. (2019, 26 de abril). WhatsApp recula (otra vez): el cierre de la cuenta de Podemos es ahora permanente. https://www.el- 
confidencial.com/tecnologia/2019-04-26/WhatsApp-podemoscuenta-cierre-caos_1964850/

Estudio Anual de Redes Sociales 2019. (2019, 5 de junio). https://iabspain.es/estudio/estudio-anual-de-redes-sociales-2019/

Estudio Datafolha. (2018). Eleições 2018. http://media.folha.uol.com.br/da tafolha/2018/10/27/44cc2204230d2fd45e18b039ee8c07a6.pdf

Financial Times. (2019). WhatsApp is a dark version of democracy. https:// www.ft.com/content/3b55465e-6039-11e9-b285-3acd5d43599e

Galarraga Gortázar, N. (2018, 29 de octubre).El ultraderechista Bolsonaro gana las elecciones y será presidente en Brasil. El País. https: // elpais.com/ internacional/2018/10/28/america/1540749476_160477.html

Giansante, G. (2015). La comunicación política online. UOC.

Gragnani, J (2018, 9 de octubre). Um Brasil dividido e movido a noticias falsas: Uma semana dentro de 272 grupos políticos no WhatsApp. Globo News. https://epocanegocios.globo.com/Brasil/ noticia/2018/10/um-brasil-dividido-e-movido-noticias-falsasuma-semana-dentro-de-272-grupos-politicos-no-WhatsApp.html

Gutiérrez-Rubí, A. (2014). Tecnopolítica: El uso y la concepción de nuevas herramientas tecnológicas para la comunicación, la organización y la acción política colectiva. Grafiko.

Gutiérrez-Rubí, A (2015). La política en tiempos de WhatsApp. El País Libros.

Gutiérrez-Rubí, A. (2019a, 11 de marzo). Internet y elecciones: Cómo las redes sociales irrumpen en la comunicación política. Infobae. https:// www.infobae.com/politica/2019/03/11/internet-y-eleccionescomo-las-redes-sociales-irrumpen-en-la-comunicacion-politica/ 
Gutiérrez-Rubí, A. (2019b, 13 de abril). La campaña oficial en WhatsApp. https://www.gutierrez-rubi.es/2019/04/13/la-campana-oficialen-WhatsApp/

Hazard, L. (2018). WhatsApp is a black box for fake news. Verificado 2018 is making real progress fixing that. http://www.niemanlab. org/2018/06/ whatsapp-is-a-black-box-for-fake-news-verificado2018-is-making-real-progress-fixing-that/

Jaume, P. (2015, 1 de abril). Hablar con los ciudadanos vía WhatsApp. La Vanguardia. https://www.lavanguardia.com/ politica/20150401/54428626261/contactar-via-WhatsApp-politicos-moda-precampana.html

Jenkins, H. (2006). Convergence Culture: Where old and new media collide. New York University Press.

Maarek, P. J. (2014). Politics 2.0: New forms of digital political marketing and political communication. Trípodos, 1(34), 13-22. http://www. tripodos.com/index.php/Facultat_Comunicacio_Blanquerna/ article/view/163

Martin, J. A. (2014). Mobile media and political participation: Defining and developing an emerging field. Mobile Media \& Communication, 2(2), 173-195.https://doi.org/10.1177/2050157914520847

Nogueira, K. (2019, 1 de enero). Na posse, bolsominions gritam "WhatsApp, WhatsApp! Facebook, Facebook!”. DCM. https://www. diariodocentrodomundo.com.br/video-na-posse-bolsominionsgritam-WhatsApp-WhatsApp-facebook-facebook/

Oliveira, J. (2018, 7 de octubre). WhatsApp, el elemento distorsionador de la campaña en Brasil. El País. https://elpais.com/internacional/2018/10/07/america/1538877922_089599.html 
Oxford Internet Institute. (2019). The Computational Propaganda Research Project (ComProp). https://comprop.oii.ox.ac.uk

Palomo Torres, M. B. y Sedano Amundarain, J. A. (2018). WhatsApp como herramienta de verificación de fake news: El caso de B de Bulo. Revista Latina de Comunicación Social, 73, 1384-1397. https://doi. org/10.4185/RLCS-2018-1312

Penteado de Camargo, C. L. (2011). Marketing político na era digital: Perspectivas e possibilidades. Revista USP, 90, 6-23. https://doi. org/10.11606/issn.2316-9036.v0i90p6-23

Senra, R. (2018, 20 de octubre). Elecciones en Brasil: Por qué WhatsApp bloqueó la cuenta del hijo de Bolsonaro y la de cientos de miles de usuarios en las vísperas de los comicios. BBC. https://www.bbc. com/mundo/noticias-america-latina-45926450

Serrano, P. (2019, 28 de febrero). La campaña política se decidirá en tu WhatsApp.Eldiario.es. https://www.eldiario.es/zonacritica/campana-politica-decidira-WhatsApp_6_872172794.html

Singh, M. (2017, 24 de febrero). WhatsApp hits 200 million active users in India.https://mashable.com/2017/02/24/whatsapp-india-200-million-active-users/

Sydow, L. (2019, 16 de enero). The State of Mobile in 2019: The Most Important Trends to Know. App Annie. https://www.appannie.com/ en/insights/market-data/the-state-of-mobile-2019/

Tardáguila, C., Benevenuto, F. y Ortellado, P. (2018, 17 de octubre). Fake News Is Poisoning Brazilian Politics. The New York Times. https:// www.nytimes.com/2018/10/17/opinion/brazil-election-fakenews-WhatsApp.html?ref=nyt-es\&mcid=nyt-es\&subid=article 
Varona-Aramburu, D., Sánchez-Martín, M. y Arrocha, R. (2017). Consumo de información política en dispositivos móviles en España: Caracterización del usuario tipo y su interacción con las noticias. El Profesional de la Información, 26(4), 641-648. https: // doi. org/10.3145/epi.2017.jul.08

Verma, L. (2017, 12 de marzo). BJP’s election strategy: 900 rallies, 67,000 workers, 10,000 WhatsApp groups and chopper landings. The Indian Express. https://indianexpress.com/elections/bjps-election-strategy-900-rallies-67000-workers-10000-whatsapp-groupschopper-landings-4565757/

Vosoyghi, S., Ray, D. y Aral, S. (2018). The Spread of True and False News Online.http://ide.mit.edu/sites/default/files/publications/2017\%20 IDE \%20Research \%20Brief \%20False \%20News.pdf

20minutos. (2019, 24 de febrero). WhatsApp cumple 10 años con 25 millones de usuarios "enganchados". https://www.20minutos.es/ noticia/3571703/0/WhatsApp-cumple-diez-anos-con-25-millones-espanoles-enganchados/ 